\title{
Smad1 promotes colorectal cancer cell migration through Ajuba transactivation
}

\author{
Daming Yang ${ }^{1, *}$, Tieying Hou ${ }^{3, *}$, Lei $\mathrm{Li}^{4}$, Yimin Chu ${ }^{1}$, Fengli Zhou ${ }^{1}$, Ying Xu $^{1}$, Xinyu \\ Hou $^{5}$, Huan Song ${ }^{6}$, Kai Zhu', Zhaoyuan Hou ${ }^{1,2,5}$, Haixia Peng ${ }^{1}$ and Hao Jia ${ }^{1,2,5}$ \\ ${ }^{1}$ Digestive Endoscopy Center, Tongren Hospital, Shanghai Jiaotong University School of Medicine, Shanghai 200336, China \\ ${ }^{2}$ Hongqiao Institute of Medicine, Tongren Hospital/Faculty of Basic Medicine, Shanghai Jiaotong University School of Medicine, \\ Shanghai 200025, China \\ ${ }^{3}$ Department of Clinical Laboratory, Guangdong General Hospital, Guangdong Academy of Medical Sciences, Guangzhou \\ 510080, China \\ ${ }^{4}$ Department of Thoracic Surgery, Lanling People's Hospital, Lanling County, Linyi 277700, China \\ ${ }^{5}$ Shanghai Key Laboratory for Tumor Microenvironment and Inflammation, Department of Biochemistry \& Molecular Cellular \\ Biology, Shanghai Jiaotong University School of Medicine, Shanghai 200025, China \\ ${ }^{6}$ Department of Gastroenterology, Ruijin Hospital, Shanghai Jiaotong University School of Medicine, Shanghai 200025, China \\ ${ }^{7}$ Liver Cancer Institute, Zhongshan Hospital, Fudan University, Shanghai 200032, China \\ * These authors have contributed equally to this work \\ Correspondence to: Haixia Peng, email: haixiapeng@163.com \\ Hao Jia, email: fonney@sjtu.edu.cn
}

Keywords: Smad 1; Ajuba; migration; colorectal cancer; Snail

Received: April 04, $2017 \quad$ Accepted: November 17, $2017 \quad$ Published: November 30, 2017

Copyright: Yang et al. This is an open-access article distributed under the terms of the Creative Commons Attribution License 3.0 (CC BY 3.0), which permits unrestricted use, distribution, and reproduction in any medium, provided the original author and source are credited.

\section{ABSTRACT}

SMAD family member 1 (Smad1) have been involved in metastatic progression of many cancer types. However, the detailed molecular signalling pathway underlying the regulatory link between Smad1 and metastasis remains elusive. Here, we demonstrate that Smad1 promotes migration of colorectal cancer (CRC) cells by inducing Snail and Ajuba expression simultaneously, but no apparent effect on Twist1 expression. Remarkably, E-cadherin, the best known Snail/Ajuba target gene is downregulated by Smad1 expression. Further, depletion of Ajuba in HCT116 cells significantly dampens the cell migration capability induced by Smad1 overexpression, suggesting that Ajuba is required for Smad1 to induce cell migration. Moreover, clinical analysis shows a significant positive correlation between the level of Smad1 and Ajuba in CRC samples. Together, our data provides the first evidence of the regulatory network of Smad1/ Snail/Ajuba axis in CRC migration, suggesting that Smad1 and Ajuba are potential new therapeutic targets and prognostic factors for CRC.

\section{INTRODUCTION}

SMA and mother against decapentaplegic (MAD)related proteins (SMADs) are intracellular components of TGF- $\beta$ signaling pathway [2]. So far, there are eight members in SMAD family, designated as Smad1-Smad8 respectively. According to the structure and function, SMADs are further divided into subgroups: receptorregulated SMADs (R-Smad including Smads1/2/3/5/8), a common SMAD (Co-Smad containing Smad4 only), and inhibitory SMADs (I-Smads) [3, 4, 19, 21]. For R-Smads, Smad1/5/8 transduce bone morphogenetic protein (BMP) signals, whereas $\operatorname{Smad} 2 / 3$ generally mediate TGF $\beta$ signaling pathway $[12,17]$.

Dysregulation of Smad functions are linked to various types of developmental defects and diseases. For example, Smad1 can be induced by many tumorstimulating cytokines such as the bone morphogenetic 
protein 2 (BMP2) and TNF $\alpha$ and plays important roles in cell invasion and metastasis [10, 20, 23]. Additionally, Smad1 is upregulated by B7-H3 via PI3K-Akt pathway and promotes Epithelial-Mesenchymal Transition (EMT) process in the colorectal cancer cells [9]. Moreover, Smad1 is identified as a target of miR-26b-5p in hepatocellular carcinoma metastasis and EMT [22]. These observations suggest a link between Smad1 and EMT. However, the detailed molecular signaling pathway underlying the regulatory link between Smad1 and cell migration and EMT remains elusive.

The Snail transcription factor is a master regulator of EMT and promotes cell migration during normal development and tumor metastases. Previous studies showed transcriptional repression and EMT induction by Snail requires recruiting Ajuba as a co-repressor [1]. Ajuba is a scaffold protein, belonging to the Zyxin/Ajuba family characterized by two or three tandem C-terminal LIM domains and a unique $\mathrm{N}$-terminal region designated as the preLIM region [5]. Ajuba is involved in many cellular processes such as cell-cell adhesion, gene transcription, cell proliferation, cell migration, and cytokinesis $[1,6]$. Several studies also suggest that Ajuba promotes EMT in colorectal cancer [15]. Most recently, we found that Ajuba is markedly upregulated in CRC and promotes CRC development by inhibiting cell apoptosis [8]. However, how the expression of Ajuba is regulated in CRC cells remain undefined.

In the present study, we demonstrate that Smad1 induces the expression of Ajuba and Snail to promote cell migration in colorectal cancer cells. Clinically, the level of Smad1 in CRC specimens is positively correlated with the levels of Ajuba. The present work provides the first evidence of the regulatory network of Smad1-Ajuba axis in CRC, suggesting that Smad1 and Ajuba are potential new therapeutic targets and prognostic factors for CRC.

\section{RESULTS}

\section{Smad1 promotes cell migration of colorectal cancer cells}

To determine whether Smad1 regulates cell migration in colon cancers, Smad1 was depleted or overexpressed in HCT116/Sw1116 cells using Smad1 siRNA/shRNA or PCDNA3.0-Smad1-expressing plasmid, the protein and the mRNA level of Smad1 were examined by western-blot and qRT-PCR assays (Figures 1A, 1D, 2A, 2D, Supplementary Figure 1A-1D). The migratory capability of the resulting cells was examined by transwell and scratching assays. Depletion of Smad1 resulted in significantly reduced migratory capacities as compared with control cells (Figure 1B, 1C, 1E). In contrast, ectopic expression of Smad1 in HCT116/Sw1116 cells enhanced cell migration (Figure 2B, 2C, 2E). We next performed CCK-8 assays to examine the effect of Smad1 on the overall cell growth. Notably, depletion or stable expression of Smad1 in HCT116 cells was not affect the cell growth (Supplementary Figure 2). Together, these data indicate that Smad1 is essential for migratory capability of HCT116 cells.

\section{Ectopic expression of Smad1 induces Snail and Ajuba expression in HCT116 cells}

We first examined the cellular morphology of three cell lines with different expression levels of Smad1 (SW1116-Mock, sh-Smad1, Smad1-OE). A distinct morphological difference was observed in those cell lines (Figure 3A). To determine if Smad1 is able to increase the expression of the known cell migration inducers such as Twist1, Snail and its cofactor Ajuba as well as EMT markers E-cadherin and N-cadherin, total RNAs and protein were extracted from HCT116-vector, HCT116Smad1, Si-Smad1 cells or Si-Ctrl were prepared. qRTPCR and Western-blot assays were performed to examine the expression of the chosen genes. Strikingly, ectopic expression of Smad1 markedly increased the mRNA and protein level of Snail and Ajuba, and depletion of Smad1 decreased the mRNA and protein level of Snail (Figure 3B, 3G) and Ajuba (Figure 3C, 3G, 3H and Supplementary Figure 1E, 1F), while the mRNA and protein level of Twist1 remained unchanged (Figure 3F, $3 \mathrm{G}, 3 \mathrm{H}$ and Supplementary Figure 1E, 1F). Remarkably, E-cadherin (Figure 3D, 3G, 3H and Supplementary Figure 1E, 1F), the best known Snail/Ajuba target gene was downregulated by Smad1 overexpression and was induced by Smad1 depletion. Conversely, the expression of N-cadherin showed opposite pattern to Snail and Ajuba (Figure 3F, 3G, 3H and Supplementary Figure 1E, 1F). To validate if the expression of Ajuba was affected by BMP2, we treated HCT116 cells and MEFs cells with bmp2 for $12 \mathrm{~h}$ and the total RNAs were extracted from the resulting cells for qRT-PCR analysis. Surprisingly, the expression of Ajuba was not apparently affected by BMP2 treatment (Figure 3I and Supplementary Figure 1G). We also had added the p-Smad1 as the positive control to prove the Ajuba was not regulated by Bmp2 (Figure 3I). Collectively, these observations suggest that Smad1 regulate EMT by transactivating Snail/Ajuba expression in HCT116 cells.

\section{Ajuba is required for Smad1 to increase cell migration in HCT116 cells}

To determine the importance of upregulation of Ajuba for Smad1 to regulate cell migration, we stably introduced vector containing shRNA specifically targeting Ajuba via viral infections into Smad1 overexpression 
HCT116 cells (Figure 4A and Supplementary Figure 1H). Remarkably, depletion of Ajuba in HCT116-Smad1 cells significantly changed cell morphology (Figure 4B), inhibited the cell migration examined by transwell and scratching assays (Figure 4C and 4D). These results, taken together, indicate that Ajuba is required for Smad1 to induce cell migration.

\section{Smad1 is positively correlated with Ajuba expression in colorectal cancer samples}

To evaluate the clinical relevance of Smad1 and Ajuba, we performed qRT-PCR assays on 40 paired CRC specimens. Consistent with previous observations, the expression of Smad1 was significantly higher in tumor compared with

A

B

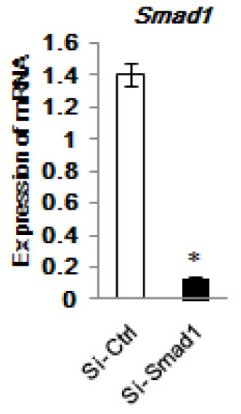

C

Si-Ctrl

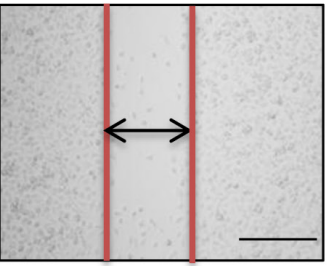

HCT116
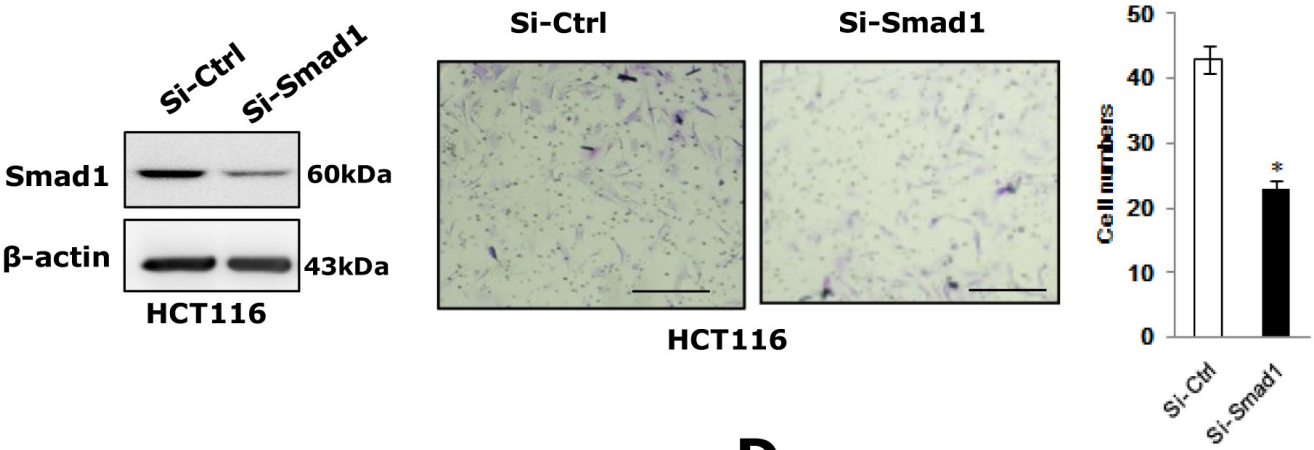

D
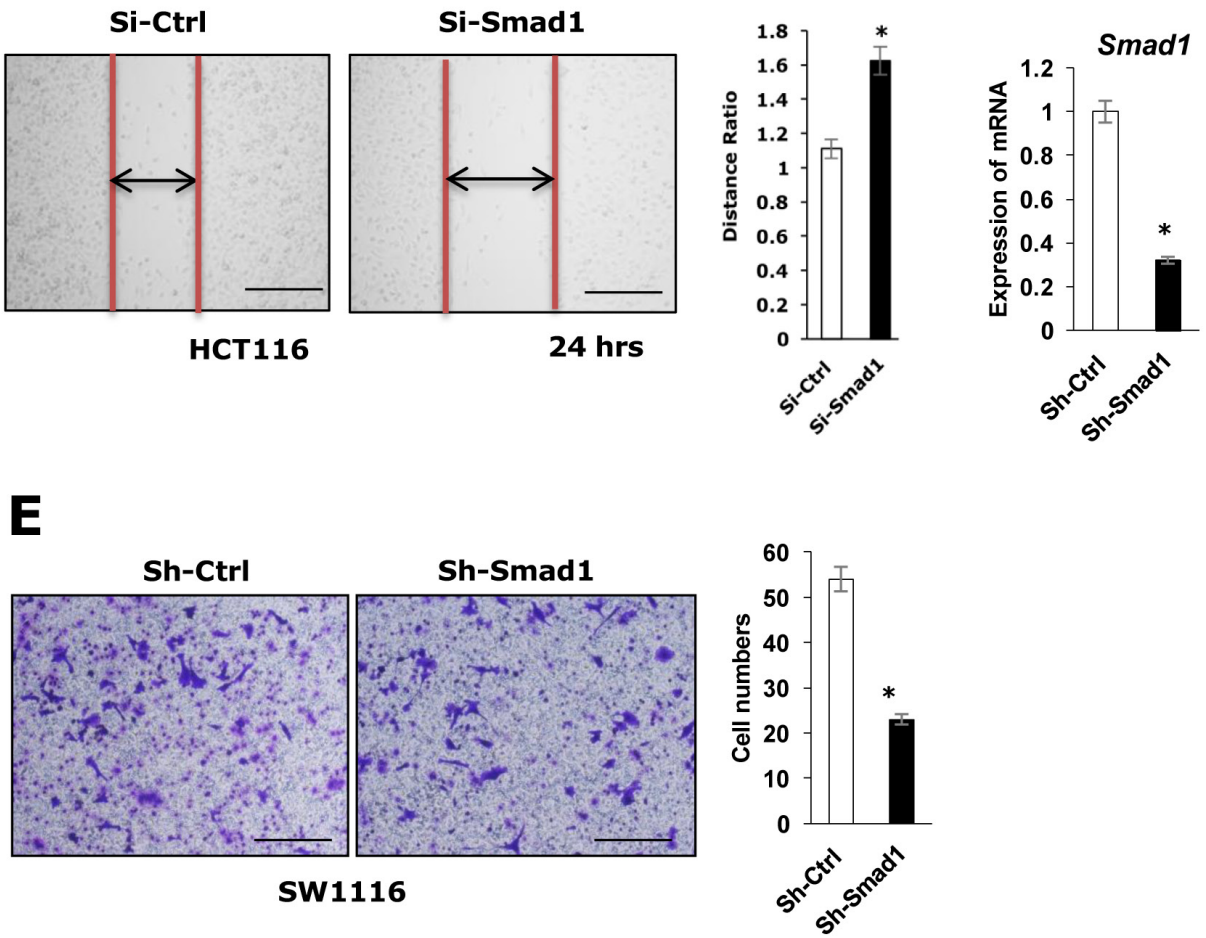

Figure 1: Knocking-down of Smad1 decreases CRC cell migration. (A) Left panel: The mRNA expression of Smad1 in HCT116 cell lines (Si-Ctrl, Si-Smad1) were shown. Data were shown as mean \pm S.D. from three independent experiments, ${ }^{*} \mathrm{p}<0.05(\mathrm{n}=3$ ). Right panel: The protein expression of Smad1 in HCT116 cell lines (Si-Ctrl, Si-Smad1) were shown. (B) Left panel: The migration ability of HCT116 cells was measured by transwell assay, the assays were repeated three times in triplicate. Right panel: Statistics were showed with bar graph. Data were shown as mean \pm S.D. from three independent experiments, ${ }^{*} \mathrm{p}<0.05(\mathrm{n}=3)$. (C) Left panel: The motility of HCT116 cells was determined by scratching assay and statistics were showed with bar graph. Right panel: Data were shown as mean \pm S.D. from three independent experiments, ${ }^{*} \mathrm{p}<0.05(\mathrm{n}=3)$. (D) Left panel: The mRNA expression of Smad1 in SW-1116 cell lines (Sh-Ctrl, Sh-Smad1) were shown. Data were shown as mean \pm S.D. from three independent experiments, ${ }^{*} \mathrm{p}<0.05(\mathrm{n}=3)$. Right panel: The protein expression of Smad1 in SW-1116 cell lines (Sh-Ctrl, Sh-Smad1) were shown. (E) Left panel: The migration ability of SW-1116 cells was measured by transwell. This assays were repeated three times in triplicate. Right panel: Statistics were showed with bar graph. Data were shown as mean \pm S.D. from three independent experiments, ${ }^{*} \mathrm{p}<0.05(\mathrm{n}=3)$. 
the para-tumor samples (Figure 5A and 5B). To examine the protein level of Ajuba and Smad1 in CRC specimens, we performed immunohistological chemistry (IHC) assays on tumour tissues (Supplementary Figure 3). Interestingly, Ajuba showed parallel expression pattern with Smad1 (Figure 5C and 5D). Pearson's correlation analysis showed that a significant positive correlation between the level of Smad1 and Ajuba in CRC samples (Figure 5E and 5F).

\section{DISCUSSION}

Colorectal cancer is the third common cancer in men and the second in Women in worldwide [18]. However, the molecular mechanisms of tumorigenesis and migration of CRCs remain largely unclear. In this paper, we demonstrate that Smad1 promotes cell migration of

A

B
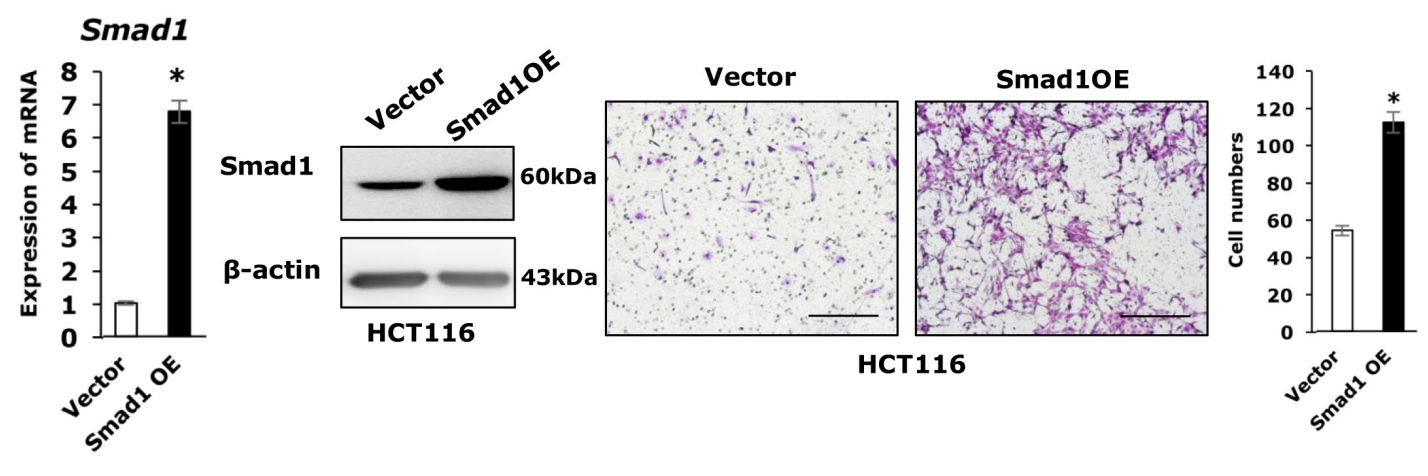

C

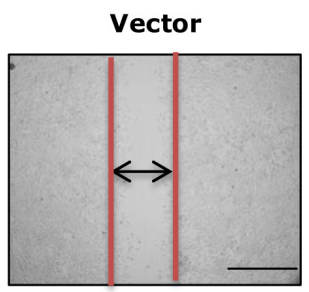

HCT116

E

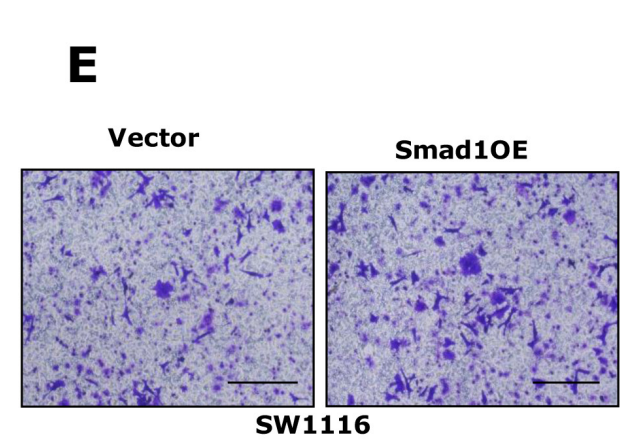

Smad10E

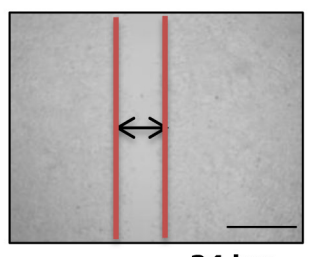

24 hrs

HCT116

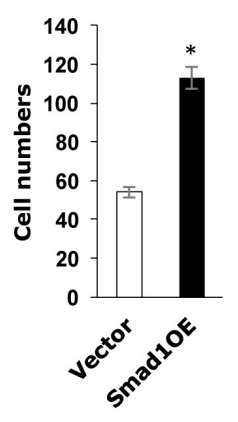

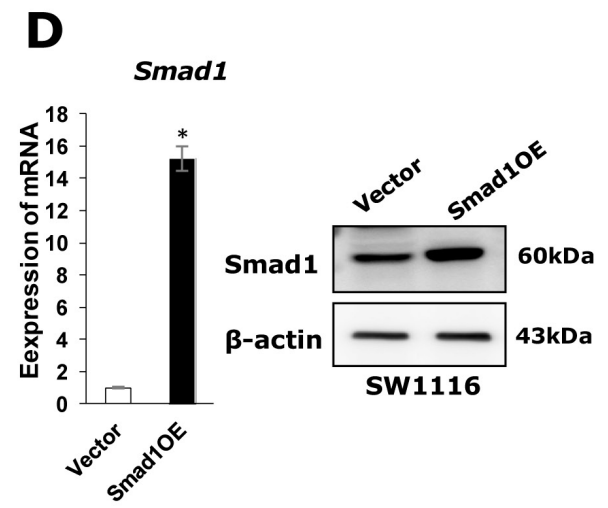

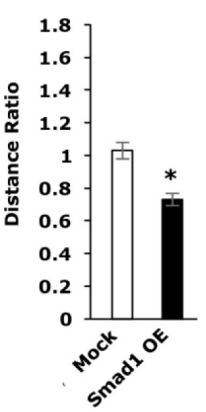

Figure 2: Smad1 promotes CRC cell migration. (A) Left panel: The mRNA expression of Smad1 in HCT116 cell lines (Vector, Smad1 OE) were shown. Data were shown as mean \pm S.D. from three independent experiments, ${ }^{*} \mathrm{p}<0.05(\mathrm{n}=3)$. Right panel: The protein expression of Smad1 in HCT116 cell lines (Vector, Smad1 OE) were shown. (B) Left panel: The migration ability of HCT116 cells was measured by transwell assay, the assays were repeated three times in triplicate. Right panel: Statistics were showed with bar graph. Data were shown as mean \pm S.D. from three independent experiments, ${ }^{*} \mathrm{p}<0.05(\mathrm{n}=3)$. (C) Left panel: The motility of HCT116 cells was determined by scratching assay and statistics were showed with bar graph. Right panel: Data were shown as mean \pm S.D. from three independent experiments, " $\mathrm{p}<0.05(\mathrm{n}=3)$. (D) Left panel: The mRNA expression of Smad1 in SW-1116 cell lines (Vector, Smad1 OE) were shown. Data were shown as mean \pm S.D. from three independent experiments, ${ }^{*} \mathrm{p}<0.05(\mathrm{n}=3)$. Right panel: The protein expression of Smad 1 in SW-1116 cell lines (Vector, Smad1 OE) were shown. (E) Left panel: The migration ability of SW-1116 cells was measured by transwell. This assays were repeated three times in triplicate. Right panel: Statistics were showed with bar graph. Data were shown as mean \pm S.D. from three independent experiments, ${ }^{*} \mathrm{p}<0.05(\mathrm{n}=3)$. 

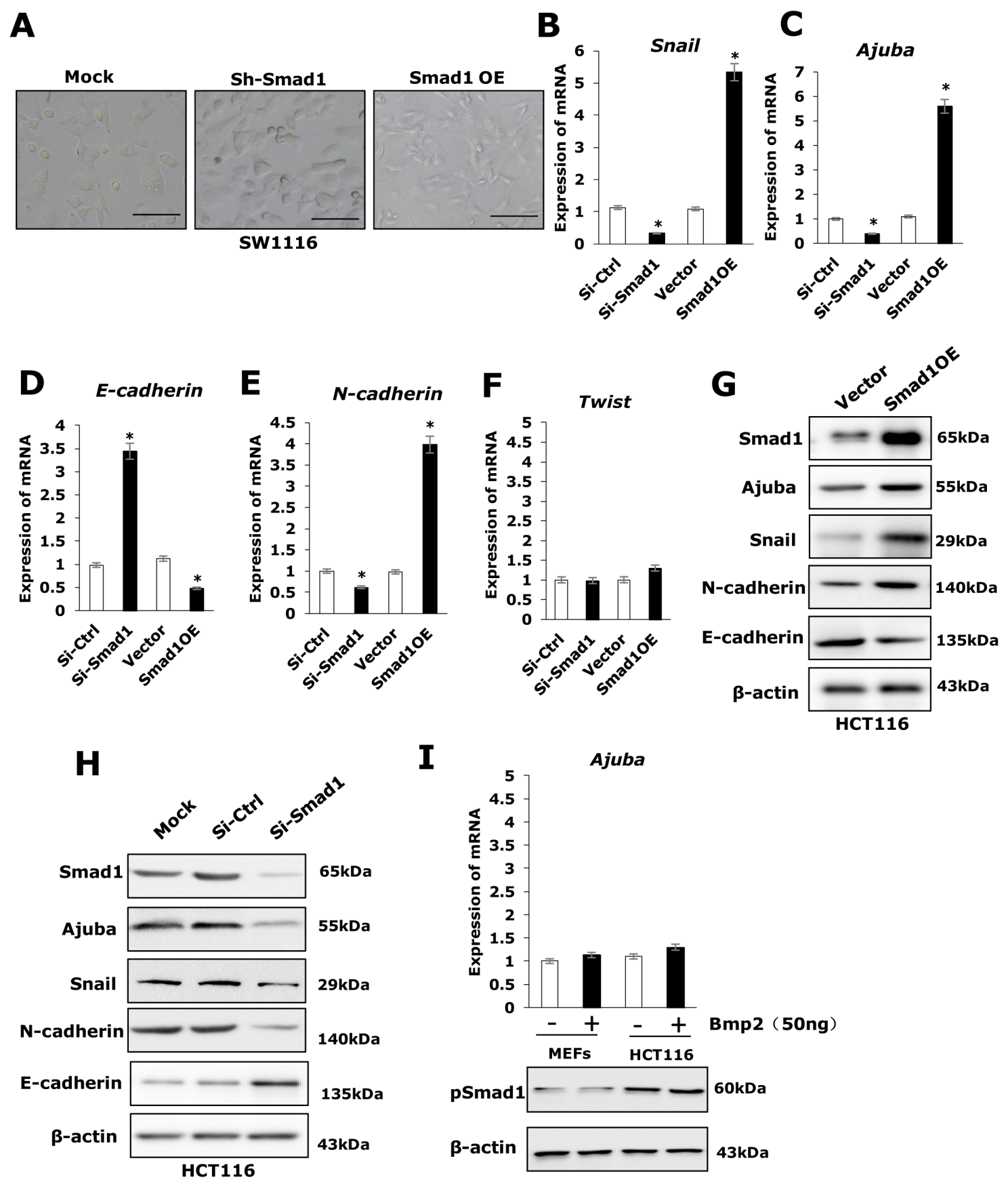

Figure 3: Smad1 promotes migration of HCT116 by enhancing EMT. (A) The cellular morphology of three cell lines (SW1116Mock, SW1116-shSmad1, and SW1116-Smad1) is shown. (B) The mRNA expression of epithelial and mesenchymal markers, Snail, as well as the transcription factors, was compared between HCT116 cell lines (Si-Ctrl, Si-Smad1, Vector, Smad1OE) by qRT-PCR. Data were shown as mean \pm S.D. from three independent experiments, ${ }^{*} \mathrm{p}<0.05(\mathrm{n}=3)$. (C) The mRNA expression of Ajuba was compared between HCT116 cell lines (Si-Ctrl, Si-Smad1, Vector, Smad1OE) by qRT-PCR. Data were shown as mean \pm S.D. from three independent experiments, ${ }^{*} \mathrm{p}<0.05(\mathrm{n}=3)$. (D) The expression of epithelial and mesenchymal markers, E-cadherin, as well as the transcription factors, was compared between HCT116 cell lines (Si-Ctrl, Si-Smad1, Vector, Smad1OE) by qRT-PCR. Data were shown as mean \pm S.D. from three independent experiments, ${ }^{*} \mathrm{p}<0.05(\mathrm{n}=3)$. (E) The expression of epithelial and mesenchymal markers, N-cadherin, as well as the transcription factors, was compared between HCT116 cell lines (Si-Ctrl, Si-Smad1, Vector, Smad1OE) by qRT-PCR. Data were shown as mean \pm S.D. from three independent experiments, ${ }^{*} \mathrm{p}<0.05(\mathrm{n}=3)$. (F) The expression of epithelial and mesenchymal markers, Twist, as well as the transcription factors, was compared between HCT116 cell lines (Si-Ctrl, Si-Smad1, Vector, Smad1OE) by qRT-PCR. Data were shown as mean \pm S.D. from three independent experiments, ${ }^{*} \mathrm{p}<0.05(\mathrm{n}=3)$. (G-H) Western blots show the EMT protein in HCT116 cells and the derivatives. (I) Up: Bmp2 did not induces Ajuba in MEFs and Hct116 cells. The cells were treated with $50 \mathrm{ng} / \mathrm{ml} \mathrm{Bmp2} \mathrm{or} \mathrm{PBS.} \mathrm{After12}$ hrs, the total RNAs were extracted from the resulting cells for qRT-PCR analysis. Data were shown as mean \pm S.D. from three independent experiments, ${ }^{*} \mathrm{p}<0.05(\mathrm{n}=3)$. Down: Western blots show the pSmad1 protein in HCT116 cells and MEFs. 

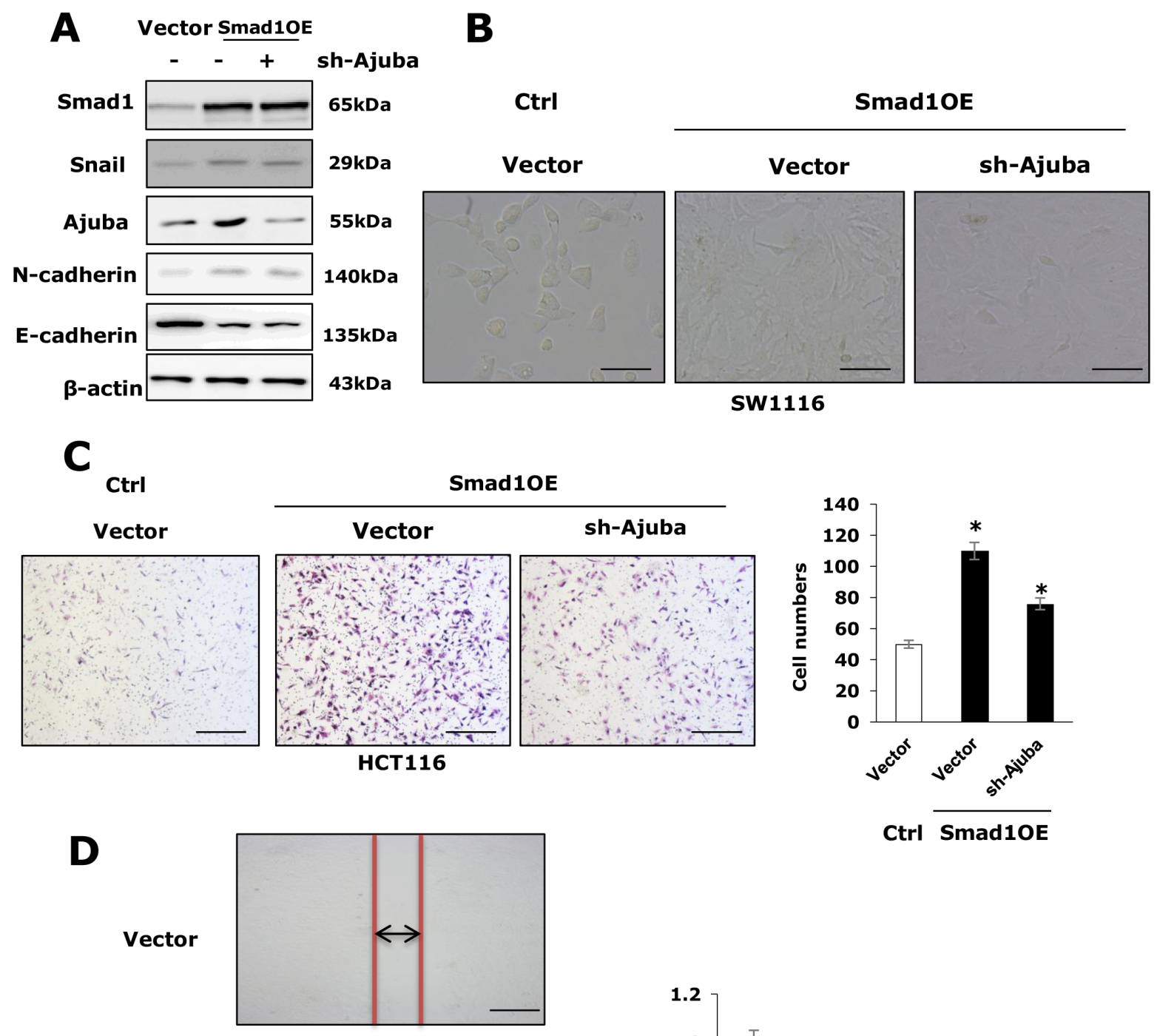

Ctrl Smad10E
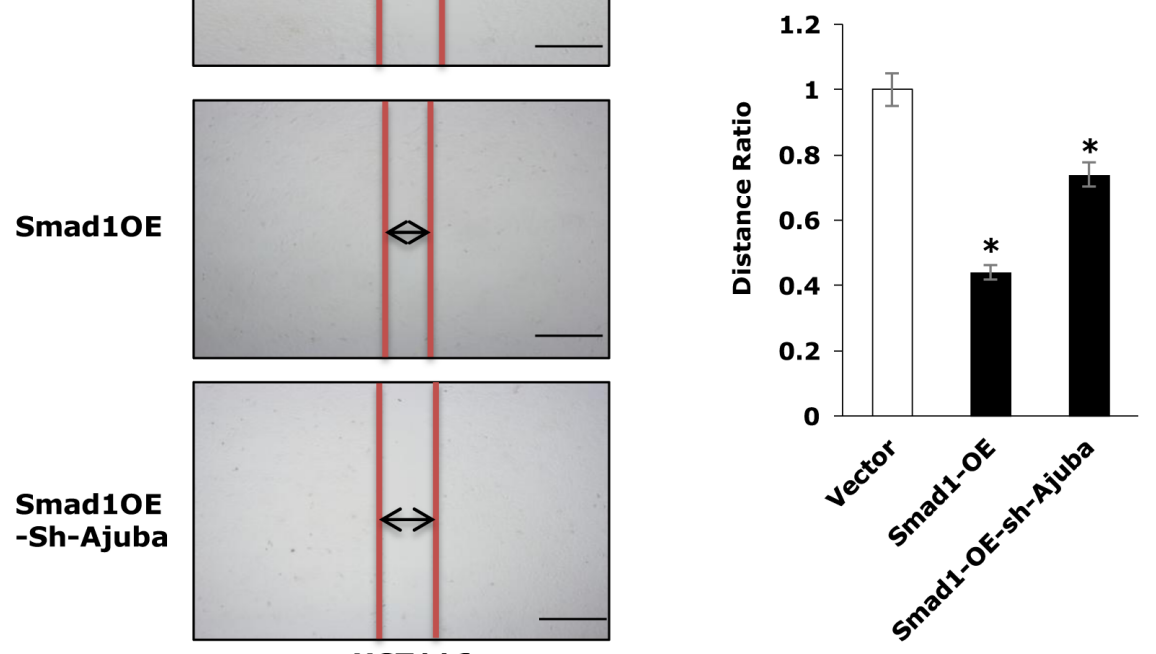

Figure 4: Ajuba was involved in cell migration and EMT induced by Smad1 deficiency in HCT116 cells. (A) Depletion of Ajuba in Smad1 over expression HCT116 cells. Western blots show the Ajuba protein in HCT116 cells and the derivatives. (B) The cellular morphology of three cell lines (SW1116-Vector, SW1116-Smad1, and SW1116-Smad1-shAjuba) is shown. (C) Left panel: Transwell assays showed repressing of Ajuba in Smad1 overexpression cells decreases cell migration in vivo compared with Smad1 overexpression group. Right panel: Data were shown as mean \pm S.D. from three independent experiments, ${ }^{*} p<0.05(n=3)$. (D) Left panel: Scratch assays showed repressing of Ajuba in Smad1 overexpression cells decreases cell migration in vivo compared with Smad1 overexpression group. Right panel: Data were shown as mean \pm S.D. from three independent experiments, ${ }^{*} \mathrm{p}<0.05(\mathrm{n}=3)$. 
colorectal cancer cells by upregulating Snail and Ajuba. Snail and Ajuba have been shown to form into a functional multi-protein complex to induce EMT and migration via transcriptional repression in various types of tumors (Figure 6). Moreover, the expression of Ajuba and Smad1 in colorectal cancer are positively correlated, suggesting that Smad1 and Ajuba may be potential therapeutic targets and prognostic factors for CRC.

The association of Smad1 with advanced cancer stage and migration are well documented. The expression of Smad1 in CRC patients have been reported by several groups in Oncomine database (https://www.oncomine. org). A series of studies also indicated that Smad1 is a critical inducer of the EMT process. PDGF-AA promotes mesenchymal stem cells migration via the BMPSmad1/5/8-Twist1/Atf4 axis and Twist1 plays the role as a downstream factor of Smad1 [13]. However, our data showed that ectopic expression of Smad1 in HCT116 increases did not increase the expression of Twist1, instead, markedly induced Snail/Ajuba expression. Snail is well known as an critical EMT inducer and promotes metastatic and tumorigenic abilities in various types of cancers [11]. Ajuba functions as an obligate co-repressor for Snail and is essential for Snail-mediated breast cancer cell migration by recruiting PRMT5 to modulate histone modifications. A recent study also indicates that an
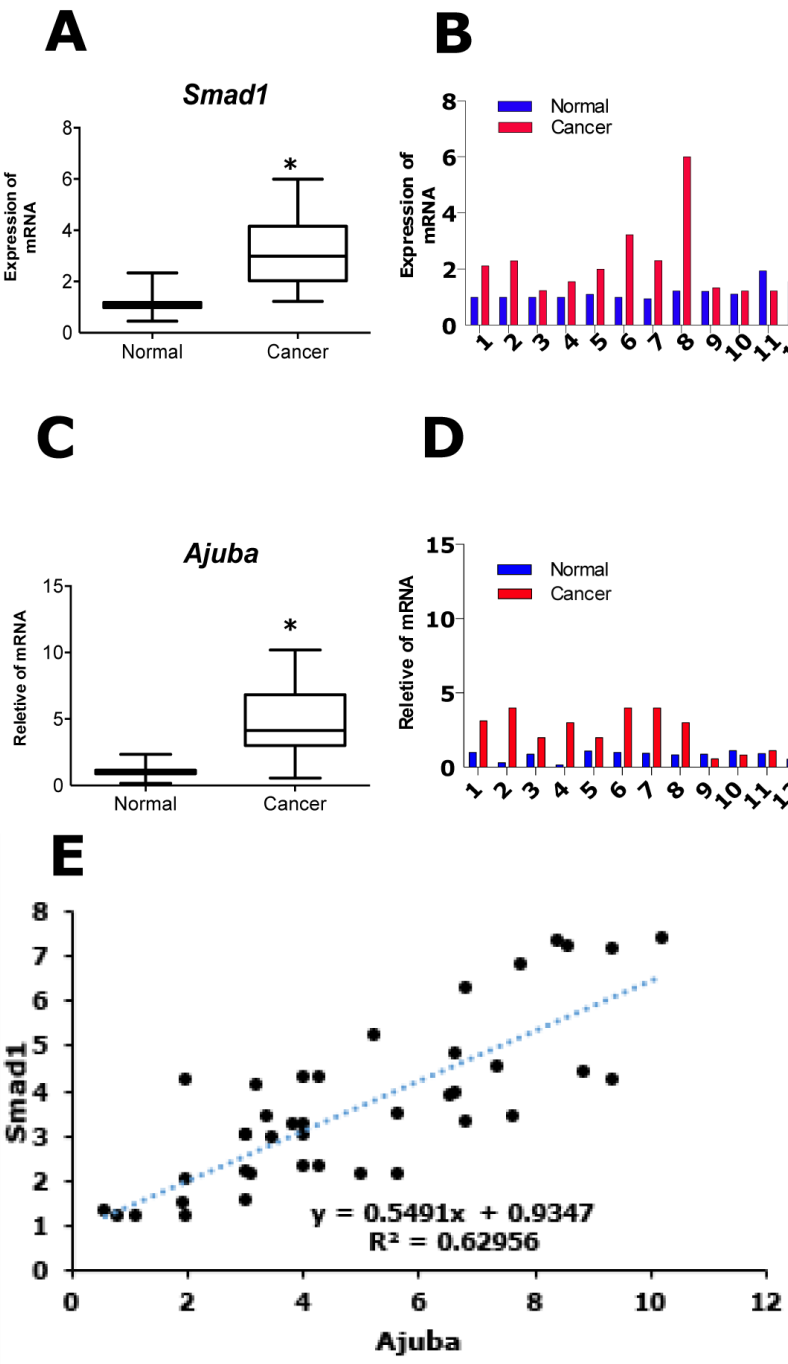

Normal

D
Smad1

Cancer

$\left.\right|_{2} ^{2}$

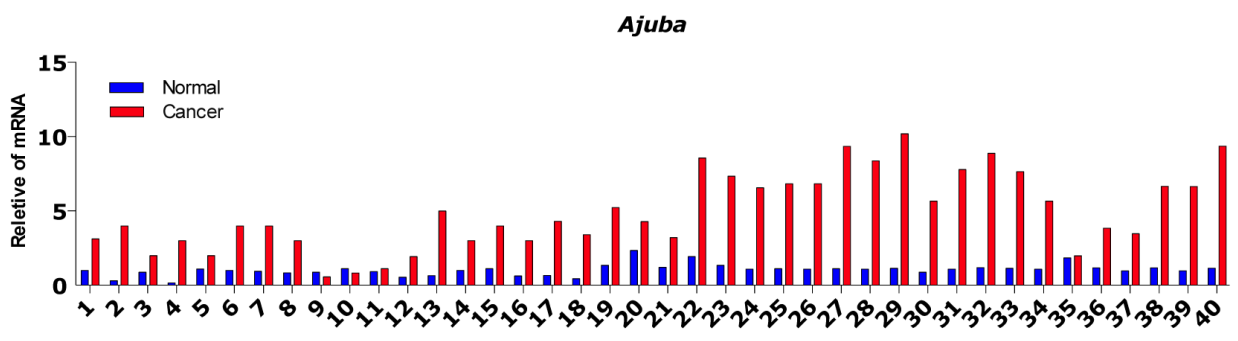

$\mathbf{F}$

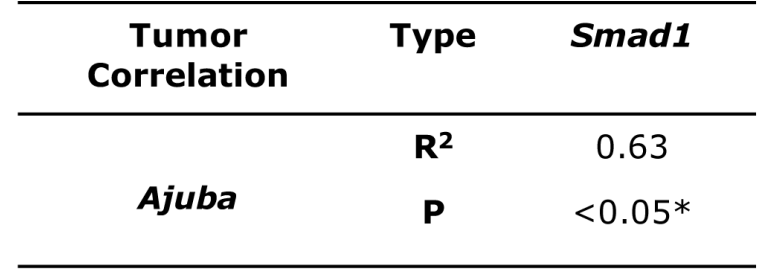

Figure 5: Clinical correlation of Smad1 in CRC patients. (A-B) The mRNA expression of Ajuba in human CRC tissues and pericancerous normal tissues was compared by qPCR ( $n=40$, paired t-test). Data were shown as mean \pm S.D. from three independent experiments, ${ }^{*} \mathrm{p}<0.05(\mathrm{n}=3)$. (C-D) The mRNA expression of Smad1 in human CRC tissues and peri-cancerous normal tissues was compared by qPCR $\left(\mathrm{n}=40\right.$, paired $\mathrm{t}$-test). Data were shown as mean \pm S.D. from three independent experiments, ${ }^{*} \mathrm{p}<0.05(\mathrm{n}=3)$. (E) Correlation analysis shows that there exists a significant positive correlation between Ajuba and Smad1 in CRC samples. (F) Pearson's correlation analysis shows that there exists a significant correlation between Ajuba and Smad1 in CRC samples. 
elevated expression of Ajuba in $\mathrm{CRC}$ may contribute to the tumor metastasis by acting as a co-repressor of Snail [15]. Interestingly, a recent study showed that Smad1 as an upstream factor regulates Snail induced PI-3 kinase/Akt and Nanog expression [16]. How Smad1 transactivates the expression of Snail remains an interesting question and need to be explored further.
In summary, our findings here highlight an important role for Smad1-Ajuba/Snail signaling in CRC cell migration [14]. The elevated of Smad1 requires the induction of the Ajuba-Snail axis but not Twist1 to promote cell migration. Disruption of the Smad1/Ajuba/ Snail axis may be potential targets for CRC therapeutics.

\section{Smad1}

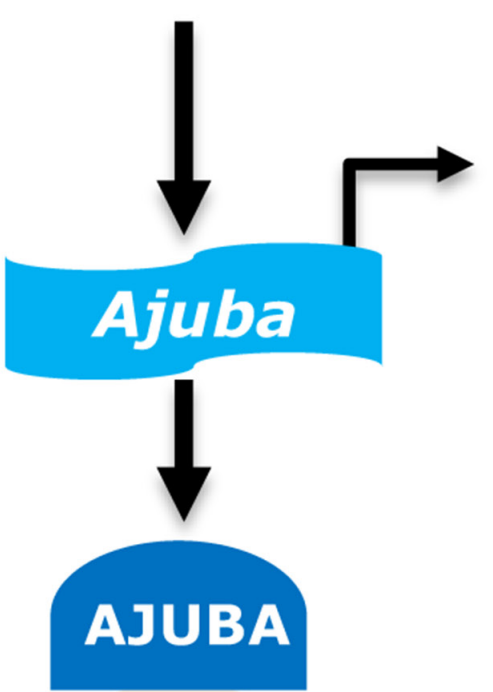

\section{Snail}

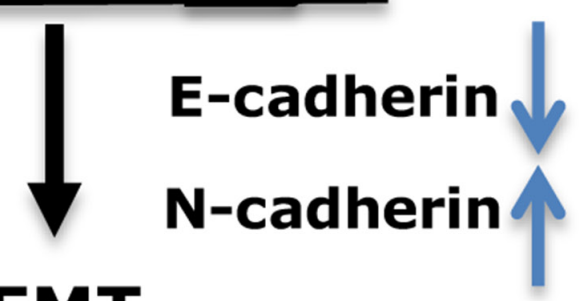

\section{EMT}

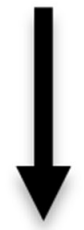

\section{Cancer}

\section{Cell migration}

Figure 6: Working model show that Smad1 may contribute to the cell migration of CRC. 


\section{MATERIALS AND METHODS}

\section{Patients and tissue samples}

A total of 40 colorectal adenocarcinoma specimens collected from patients, who received the operation at the Department of Digestive endoscopy center of the Tongren Hospital of Shanghai of China, were used in this study. All methods were carried out in accordance with relevant guidelines and regulations. All experimental protocols were approved by a shanghai jiaotong university licensing committee. Informed consent had been obtained from all patients and the project had been approved by the local Ethics Committee of Tongren Hospital of Shanghai of China.

\section{Western blot and antibodies}

Cells were lysed in buffer containing $20 \mathrm{mmol} / \mathrm{L}$ Tris- $\mathrm{HCl}$ (pH 8.0), $150 \mathrm{mmol} / \mathrm{L} \mathrm{NaCl}, 2.5 \mathrm{mmol} / \mathrm{L}$ EDTA, $0.5 \% \mathrm{NP} 40,0.1 \mathrm{mmol} / \mathrm{L}$ phenylmethylsulfonylfluoride, and protease inhibitor cocktail. Antibodies for Smad1(Cell Signaling, \#9743), Snail(Santa Cruz; sc-28199), N-cadherin (Cell Signaling, \#4061), E-cadherin (Cell Signaling, \#3195), Ajuba (Cell Signaling, \#4897) and $\beta$-Actin (Proteintech, 600081-Ig) were purchased. The assay was carried out in triplicate.

\section{Real-time PCR analysis}

Total RNA was extracted from cells with TRIzol reagent (Invitrogen) following the manufacturer's protocol. Complementary DNAs were synthesized with $2 \mu \mathrm{g}$ of total RNA using iScriptcDNA Synthesis Kit (Fermentas). The detection and quantification of target mRNA were performed with real-time PCR. The assay was carried out in triplicate. Sequence of Primers for qRT-PCR:

Smad1: F:5'-ctcatgtcatttactgccgtgtgtg-3' R: 5'-ATTCAGAAACGGTTCTTATTGTTG-3'

E-cadherin: F: 5'-TTGCTACTGGAACAGG GACAC-3' R: 5'-CCCGTGTGTTAGTTCTGCTGT-3'

N-cadherin: F: 5'-TTATCCTTGTGCTGATGT TTGTG-3' R: 5'-TCTTCTTCTCCTCCACCTTCTTC-3' Snail F: 5'-TCCAGAGTTTACCTTCCAGCA-3' R: 5'-CTTTCCCACTGTCCTACTCTG-3'

\section{Smad1 and Ajuba knockdown}

Smad1 knockdown cells were determined with the siRNAs assay as previously described [14]. For transient Smad1 knockdown, siRNAs were used (Thermo Scientific and Santa Cruz Biotechnology, Inc.). For stable knockdown of Smad1, four siRNA hairpins expressed by a retrovirus vector $(37928,31427,32418$, and 30824 , Dharmacon, Inc.) were tested. Two of them (37928 and 30824) could efficiently knock down Smadl and were used further in this study. The plasmids of PLKO.1shAjuba and shSmadl were described previously [8].

\section{Cell culture, transfection and retroviral infection}

HCT116 and 293T cells were obtained from the ATCC and were tested and authenticated by DNA typing. The cells were cultured in DMEM supplemented with $10 \%$ FBS, $2 \mathrm{~m}$ ML- glutamine and penicillin $(50 \mathrm{U} / \mathrm{ml}) /$ streptomycin $(50 \mu \mathrm{g} / \mathrm{ml})$ at $37{ }^{\circ} \mathrm{C}$ under $5 \% \mathrm{CO}_{2}$ in a humidified chamber. The transfection was performed using Lipofectamine 2000 (Invitrogen, Carlsbad, CA, USA) as described [7]. Supernatants containing viruses were packed from $293 \mathrm{~T}$ cells. When growing to 60 $80 \%$ confluence, HCT116 cells were infected with viral supernatants, and $5 \mu \mathrm{g} / \mathrm{ml}$ puromycin was added to select the stable cells.

\section{Transwell cell migration assays}

Cells were harvested after serum-free starvation for $12 \mathrm{hrs}$, and were resuspended in plain DMEM media. Ten thousand cells were applied to 8 - $\mu \mathrm{m}$ pore transwell filters (Corning). DMEM media containing 10\% FBS were added to the bottom chamber as attractants. After incubation for $24 \mathrm{hrs}$, the nonmigrated cells at the top of the filter were removed and the migrated cells at the bottom of the filter were fixed with $4 \%$ paraformaldehyde and were stained with colloidal staining method. The number of migrating cells in each chamber was quantified by counting nine randomly chosen fields under $\times 20$ magnification using a bright-field microscope. Each condition was performed in duplicate, and the average number of cells per field was represented. Experiments were repeated three times.

\section{Scratch assays}

For the scratch assay, cells were grown to confluence in a 24-wells plate, and a "wounding" line was scratched into the cell monolayer with a sterile $200-\mathrm{mL}$ pipette tip. The width of the wound was measured under a microscope at 0 and $24 \mathrm{hrs}$ after the scratch to assess the migration ability of the cells.

\section{CCK-8 assays}

Cells were aliquoted into a 96-well plate (2000 cells in $200 \mu \mathrm{l}$ per well) and were incubated for $24 \mathrm{hrs}$, $48 \mathrm{hrs}, 72 \mathrm{hrs}$ and $96 \mathrm{hrs}$. Twenty microliters of cck- 8 bromide solution were added at the indicated time point, and the aliquots were incubated for $1 \mathrm{hrs}$. One hundred fifty microliters of dimethyl sulfoxide were replaced with 200ul DMEM that contained $10 \%$ FBS, and the aliquots were shaken for 10 minutes. Absorbance at $490 \mathrm{~nm}$ was measured to determine the number of viable cells in each well. 


\section{Statistical analysis}

Each experiment was repeated at least three times with comparable results. Results are expressed as mean \pm S.D. Statistical analysis was performed using Student's two tailed t-test exact test to compare in vitro and in vivo experiments, with $\mathrm{P}$ values $<0.05$ considered statistically significant. Pearson's correlation analysis was used to determine the correlation of the expression levels of Smad1 using SPSS 13.0 software. All statistical tests were carried out using SPSS software (SPSS 13; SPSS Inc., Chicago, IL, USA).

\section{Author contributions}

Haixia Peng, Tieying Hou, Yimin Chu, Ying Xu, Lei Li, Huan Song and Hao Jia performed the experiments. Daming Yang, Fengli Zhou, Xinyu Hou and Kai Zhu analyzed the data and wrote the manuscript. Zhaoyuan Hou and Hao Jia conceived the study, supervised the project and wrote the paper.

\section{ACKNOWLEDGMENTS AND FUNDING}

We thank Dr. Baojie Li for his critical reading and insightful suggestions. This work was supported the National Science Foundation of China (81372309, 81402376, 31671415, 31601114, 81402177); Shanghai Committee of Science and Technology (15410724200); Shanghai Municipal Commission of Health and Family Planning (No. 201440426); and Science and Technology Commission of Changning District of Shanghai No. CNKW2014F01); Key Project of Science and Technology Commission of Changning District, Shanghai (No. CNKW2016Z01); Youth Foundation of Shanghai Municipal Commission of Health and Family Planning (No. 20154Y0160).

\section{CONFLICTS OF INTEREST}

Competing financial interests: The authors declare no competing financial interests.

\section{REFERENCES}

1. Ayyanathan K, Peng H, Hou Z, Fredericks WJ, Goyal RK, Langer EM, Longmore GD, Rauscher FJ 3rd. The Ajuba LIM domain protein is a corepressor for SNAG domain mediated repression and participates in nucleocytoplasmic Shuttling. Cancer Res. 2007; 67:9097-9106.

2. Canalis E, Economides AN, Gazzerro E. Bone morphogenetic proteins, their antagonists, and the skeleton. Endocr Rev. 2003; 24:218-235.

3. de Menezes EG, Machado AA, Barbosa F Jr, de Paula FJ, Navarro AM. Bone metabolism dysfunction mediated by the increase of proinflammatory cytokines in chronic HIV infection. J Bone Miner Metab. 2017; 35:234-242.

4. Gabbitas B, Canalis E. Bone morphogenetic protein-2 inhibits the synthesis of insulin-like growth factor-binding protein-5 in bone cell cultures. Endocrinology. 1995; 136:2397-2403.

5. Hou Z, Peng H, Ayyanathan K, Yan KP, Langer EM, Longmore GD, Rauscher FJ 3rd. The LIM protein AJUBA recruits protein arginine methyltransferase 5 to mediate SNAIL-dependent transcriptional repression. Mol Cell Biol. 2008; 28:3198-3207.

6. Hou Z, Peng H, White DE, Wang P, Lieberman PM, Halazonetis T, Rauscher FJ 3rd. 14-3-3 binding sites in the snail protein are essential for snail-mediated transcriptional repression and epithelial-mesenchymal differentiation. Cancer Res. 2010; 70:4385-4393.

7. Jia H, Cong Q, Chua JF, Liu H, Xia X, Zhang X, Lin J, Habib SL, Ao J, Zuo Q, Fu C, Li B. p57Kip2 is an unrecognized DNA damage response effector molecule that functions in tumor suppression and chemoresistance. Oncogene. 2015; 34:3568-3581.

8. Jia H, Song L, Cong Q, Wang J, Xu H, Chu Y, Li Q, Zhang Y, Zou X, Zhang C, Chin YE, Zhang X, Li Z, et al. The LIM protein AJUBA promotes colorectal cancer cell survival through suppression of JAK1/STAT1/IFIT2 network. Oncogene. 2017; 36:2655-2666.

9. Jiang B, Zhang T, Liu F, Sun Z, Shi H, Hua D, Yang C. The co-stimulatory molecule B7-H3 promotes the epithelial-mesenchymal transition in colorectal cancer. Oncotarget. 2016; 7:31755-31771. https://doi.org/10.18632/ oncotarget.9035.

10. Kaneda A, Fujita T, Anai M, Yamamoto S, Nagae G, Morikawa M, Tsuji S, Oshima M, Miyazono K, Aburatani H. Activation of Bmp2-Smad1 signal and its regulation by coordinated alteration of $\mathrm{H} 3 \mathrm{~K} 27$ trimethylation in Rasinduced senescence. PLoS Genet. 2011; 7:e1002359.

11. Langer EM, Feng Y, Zhaoyuan H, Rauscher FJ 3rd, Kroll KL, Longmore GD. Ajuba LIM proteins are snail/slug corepressors required for neural crest development in Xenopus. Dev Cell. 2008; 14:424-436.

12. Lawrence DA. Deficient R-smad/smad4 complex formation in fibroblasts growth-stimulated by TGF-beta 1. Int J Oncol. 2002; 20:803-806.

13. Li A, Xia X, Yeh J, Kua H, Liu H, Mishina Y, Hao A, Li B. PDGF-AA promotes osteogenic differentiation and migration of mesenchymal stem cell by down-regulating PDGFRalpha and derepressing BMP-Smad1/5/8 signaling. PLoS One. 2014; 9:e113785.

14. Li Q, Peng H, Fan H, Zou X, Liu Q, Zhang Y, Xu H, Chu Y, Wang C, Ayyanathan K, Rauscher FJ, Zhang K, Hou Z. The LIM protein Ajuba promotes adipogenesis by enhancing PPARgamma and p300/CBP interaction. Cell Death Differ. 2016; 23:158-168. 
15. Liang XH, Zhang GX, Zeng YB, Yang HF, Li WH, Liu QL, Tang YL, He WG, Huang YN, Zhang L, Yu LN, Zeng XC. LIM protein JUB promotes epithelial-mesenchymal transition in colorectal cancer. Cancer Sci. 2014; 105:660-666.

16. Liu CW, Li CH, Peng YJ, Cheng YW, Chen HW, Liao PL, Kang JJ, Yeng MH. Snail regulates Nanog status during the epithelial-mesenchymal transition via the Smad1/Akt/ GSK3beta signaling pathway in non-small-cell lung cancer. Oncotarget. 2014; 5:3880-3894. https://doi.org/10.18632/ oncotarget.2006.

17. Lopez-Rios J. Sensing BMP pathway activity by immune detection of phosphorylated R-Smad proteins in mouse embryonic kidney. Methods Mol Biol. 2012; 886:267-273.

18. Marley AR, Nan H. Epidemiology of colorectal cancer. Int J Mol Epidemiol Genet. 2016; 7:105-114.

19. Pereira RC, Rydziel S, Canalis E. Bone morphogenetic protein-4 regulates its own expression in cultured osteoblasts. J Cell Physiol. 2000; 182:239-246.
20. Ruan X, Zuo Q, Jia H, Chau J, Lin J, Ao J, Xia X, Liu H, Habib SL, Fu C, Li B. P53 deficiency-induced Smad1 upregulation suppresses tumorigenesis and causes chemoresistance in colorectal cancers. J Mol Cell Biol. 2015; 7:105-118.

21. Varghese S, Rydziel S, Canalis E. Bone morphogenetic protein-2 suppresses collagenase- 3 promoter activity in osteoblasts through a runt domain factor 2 binding site. $\mathrm{J}$ Cell Physiol. 2005; 202:391-399.

22. Wang Y, Sun B, Zhao X, Zhao N, Sun R, Zhu D, Zhang Y, Li Y, Gu Q, Dong X, Wang M, An J. Twist1-related miR-26b-5p suppresses epithelial-mesenchymal transition, migration and invasion by targeting SMAD1 in hepatocellular carcinoma. Oncotarget. 2016; 7:2438324401. https://doi.org/10.18632/oncotarget.8328.

23. Zuscik MJ, Baden JF, Wu Q, Sheu TJ, Schwarz EM, Drissi H, O'Keefe RJ, Puzas JE, Rosier RN. 5-azacytidine alters TGF-beta and BMP signaling and induces maturation in articular chondrocytes. J Cell Biochem. 2004; 92:316-331. 